\title{
Using Computer Peripheral Devices to Measure Attentiveness
}

\author{
Dalila Durães $^{1}$, Davide Carneiro ${ }^{2,3}$, Javier Bajo ${ }^{1}$, Paulo Novais ${ }^{2}$ \\ ${ }^{1}$ Department of Artificial Intelligence, Technical University of Madrid, Madrid, Spain \\ d.alves@alumnos.upm.es, jbajolfi.upm.es \\ ${ }^{2}$ Algoritmi Center, Minho University, Braga, Portugal \\ dcarneiro@di.uminho.pt, pjon@di.uminho.pt \\ ${ }^{3}$ CIICESI, ESTGF, Polytechnic Institute of Porto, Portugal
}

\begin{abstract}
Attention is strongly connected with learning and when it comes to acquiring new knowledge, attention is one the most important mechanisms. The learner's attention affects learning results and can define the success or failure of a student. The negative effects are especially significant when carrying out long or demanding tasks, as often happens in an assessment. This paper presents a monitoring system using computer peripheral devices. Two classes were monitored, a regular one and an assessment one. Results show that it is possible to measure attentiveness in a non-intrusive way.
\end{abstract}

Keywords: Attentiveness, Learning Activities, Mental Fatigue, Stress.

\section{Introduction}

Our society, in permanent change, requires a continuous adaptation of the human being to the surrounding environment. We live in a global, multicultural and hyperconnected world where technology is present in all spheres of life and is the backbone for the transformation of society.

In the education field, ICT should be considered as an innovation, since it involves a personal and collective work of reflection, realization and change. So ICT has expanded the range of possibilities of teaching and implementing innovative methodologies.

Scientific studies have shown the influence of states attention on student learning [1-3]. These indicators are useful to predict the behavior of a student and identify potential problems in the course of their learning. By using behavioral biometrics, especially Keystroke and Mouse Dynamics, it's possible analyze the type of the task performed by each user, the time spent performing it, as well as the mental workload of the task. With this information it's also possible for classifiers to distinguish situations in which each user shows signs of attentiveness and where higher or lower mental workload is measured [1].

This technique is based only on the observation of the use of the mouse and the keyboard, which allows an assessment of the user's performance. Consequently, we

adfa, p. 1, 2011.

(C) Springer-Verlag Berlin Heidelberg 2011 
considered both non-invasive and non-intrusive approaches. In this way we can use this technique to develop the attention level and management initiatives in the context of learning activities, allowing teachers to perceive the student's state of mind and adjust the teaching process to the student's needs and behavior.

Teaching should be solidly grounded to the absolute understanding of how the process of learning occurs so that instructional strategies could be efficient and lead to persistent knowledge. This is especially true when learning activities involve technologies. In such cases, some of the previously mentioned issues can negatively affect the acquisition of knowledge and the persistence of that same knowledge since students have other technologies and applications that they can use and this can distract them.

In this article we focus on a new field of application of ICT techniques and technologies in learning activities. The goal is to determine the level of attentiveness in normal and assessment lessons and compare it with results achieved by students.

This paper is organized as follows. In the next Section theoretical foundations of attention where scientific literature is reviewed, Section 3 analyzes keyboard and mouse for attentiveness detection. Finally, in Section 4 and 5 some initial results and conclusions of this work are presented.

\section{Theoretical Foundations}

The concept of attention has had different definitions since the nineteenth century. Initially, it was only a study field of psychology. However, in recent years it was object of study in different areas including biology, education science, psychiatry, and computer science.

Being a cognitive process, attention is strongly connected with learning [2]. When it comes to acquiring new knowledge, attention can be considered one of the most important mechanisms [1]. The level of the learner's attention affects learning results. The lack of attention can define the success of a student. In learning activities, attention is also very important to perform these tasks in an efficient and adequate way.

When students are using technology, and especially when connected to the Internet, distractions can occur. This happens because they have access to messages from chats, social networks and emails; and other applications like music applications and news sites, which can be more attractive to students. Moreover, these applications can constantly run in the background. When these activities are prolonged for a long period they may have decreased the level of attention [4].

\subsection{Features that influence attention}

Generally, there are some factors that influence attention level: stress, mental fatigue, anxiety, emotions, different environment and human health [16].

Stress may have a positive or negative influence. On the one hand it is generally accepted that stressful events increase the level of attention [5 - 9]. On the other hand, there are cases in which stressful events cause depression or aging [10,11]. 
When some activities are prolonged for a long period of time, our brain may feel overheaded with such amount of information, and this leads to a potential emergence of mental fatigue, which decreases the level of students' attention.

A substantial literature shows that anxiety affects perceptual and related processes of attention [12]. Anxiety has an impact on cognition and attention because it is often associated with adverse effects on attention of cognitive tasks [13].

Finally, health problems, mood, and the surrounding environment can also influence the level of attentiveness. Figure 1 presents a design of factors that influence attention.

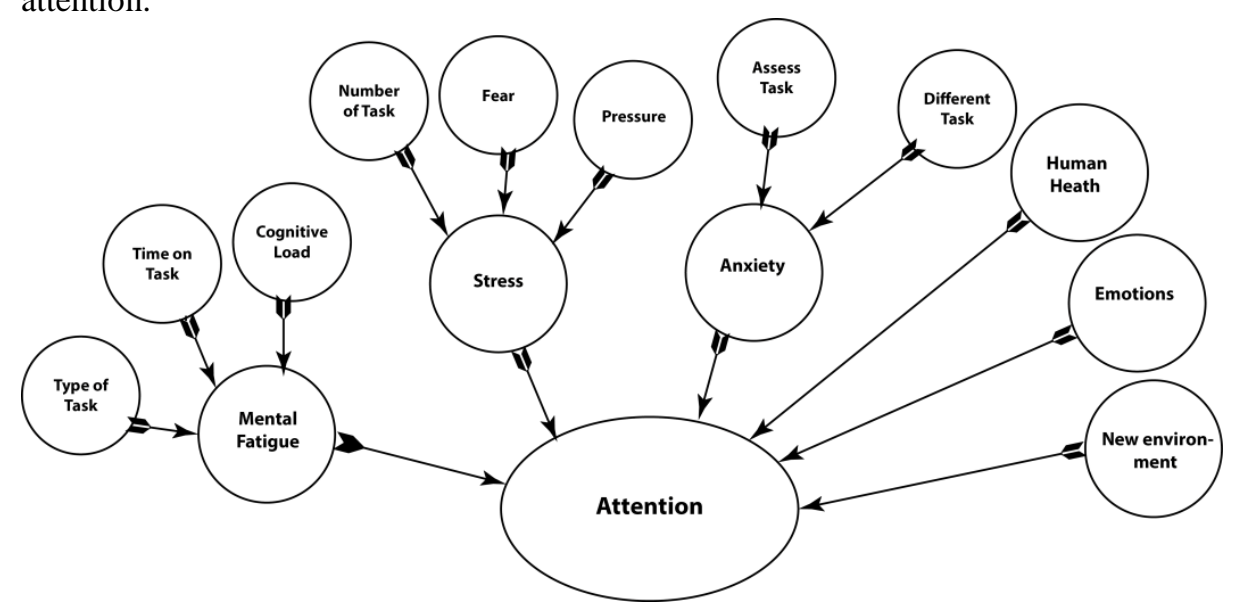

Fig. 1. Conceptualization of the set of factors that influence attention.

\section{Study Outline}

The purpose of this work is to compare a normal and an assessment class at the Secondary School of Caldas das Taipas, Guimarães, Portugal. We want to determine if classes with different goals have a significant effect on mouse and keyboard dynamics and how can we estimate attention level.

\subsection{Methodology}

For this purpose, a group of 13 (10 girls and 3 boys) art students were selected to participate, whose average age is seventeen years old. In different weeks, they have a normal and an assessment lesson, where they have access to an individual computer and three hours to complete a task. The lesson started at 8:30 and finished at 11:00 a.m. Students received, at the beginning of the lesson, a document with the goals of the task. The normal and the assessment lessons contained tasks to be completed using Photoshop.

Data collection was carried out using a logger application developed in previous work [4]. The data collected by the logger application, characterizing the students' interaction patterns, is aggregated in a server to which the logger application connects 
after the student logs in. This application runs in the background, which makes the data acquisition process, a completely transparent one, from the point of view of the student.

\subsection{Data Analysis}

In this section we show the existence of different behaviors in the two different lessons. Data was analyzed in two different ways. First was carried out a general analysis in which statically methods are used to obtain preliminary conclusions. Second, an individual analysis was made in order to compare the different moments.

Although the collected data describes the interaction with both the mouse and the keyboard [14], only data from the mouse was considered in this analysis. This is due to the characteristics of the task, which was based on Photoshop that requires mostly the interaction with the mouse The amount of data collected from the keyboard was too small to allow sound analyses. Another important aspect worth mentioning is that Photoshop requires a precise use of the mouse, which makes it a suitable application to the current study.

In a preliminary analysis of the data, we concluded that there are indeed different interaction patterns depending on the type of lesson analyzed indeed. To conclude this, we looked at the distributions of the data collected and analyzed the statistical significance of their differences. To this end, we used the Kruskal-Wallis test. Table 1 details the mean value of each feature in each class (evaluation and normal). It also details the $p$-value of the Kruskal-Wallis test.

\begin{tabular}{|l|c|c|c|c|}
\hline \multicolumn{1}{|c|}{ Feature } & Symbol & Significance & Mean Evaluation & Mean Normal \\
\hline Mouse Velocity & $\mathrm{mv}$ & 0,0011 & 0,49 & 0,53 \\
\hline Mouse Aceleration & $\mathrm{ma}$ & 0,0010 & 0,54 & 0,57 \\
\hline Click Duration & $\mathrm{cd}$ & 0,0035 & 245,38 & 159,83 \\
\hline Time Between Clicks & $\mathrm{tbc}$ & 0,0966 & 1964,19 & 3063,11 \\
\hline Distance Between Click & $\mathrm{dbc}$ & 0,0001 & 150,83 & 206,12 \\
\hline Duration Distance Clicks & $\mathrm{ddc}$ & 0,0545 & 143,04 & 143,97 \\
\hline Excess Distance Between Clicks & $\mathrm{edbc}$ & 0,0000 & 154,00 & 309,48 \\
\hline Absolute Excess Distance Between Click & aedbc & 0,0000 & 1,54 & 2,05 \\
\hline Absolute Sum Distance Between Clicks & asdbc & 0,0094 & 4006,31 & 5038,24 \\
\hline Distance Point to Line Between Clicks & dplbc & 0,0169 & 21611000,00 & 37026200,00 \\
\hline Absolute Distance Point Between Clicks & adpbc & 0,1361 & 157647,00 & 208223,00 \\
\hline
\end{tabular}

Table 1. Results of the Kruskall-Wallis test and mean values for ech class and each feature.

When data from the two classes is compared, the first conclusion is that the differences observed are statistically significant in nearly all features, with the exception of Time Between Clicks, Distance During Clicks, and Average Distance Point Between Clicks. 
Moreover, mean values of the features are consistently lower in the evaluation class. In most of the features, this indicates an increased performance (e.g. a smaller average distance between clicks means that the student moved the mouse in a more efficient manner). However, in the case of mouse velocity, for example, a smaller velocity could point out a slower, and thus less efficient, movement. In past work we concluded that a slower mouse velocity is indeed necessary for the student to achieve increased accuracy in mouse movement: moving the mouse too fast would make precise movements more difficult to carry out. This is especially true in tasks such as those of this study. A similar trend happens with mouse acceleration and click duration. The remaining features consistently show increased performance in the evaluation class.

The Figure 2 shows the distribution of the values for all the users and for each feature. We can see that the results of each feature are very different in the two lessons. The students react in different ways in the assessment and normal lesson. We can also consider that, in general, they are more focused on the assessment lesson because they have a mouse velocity, mouse acceleration, and distance point to line between clicks slower and the click duration was higher.
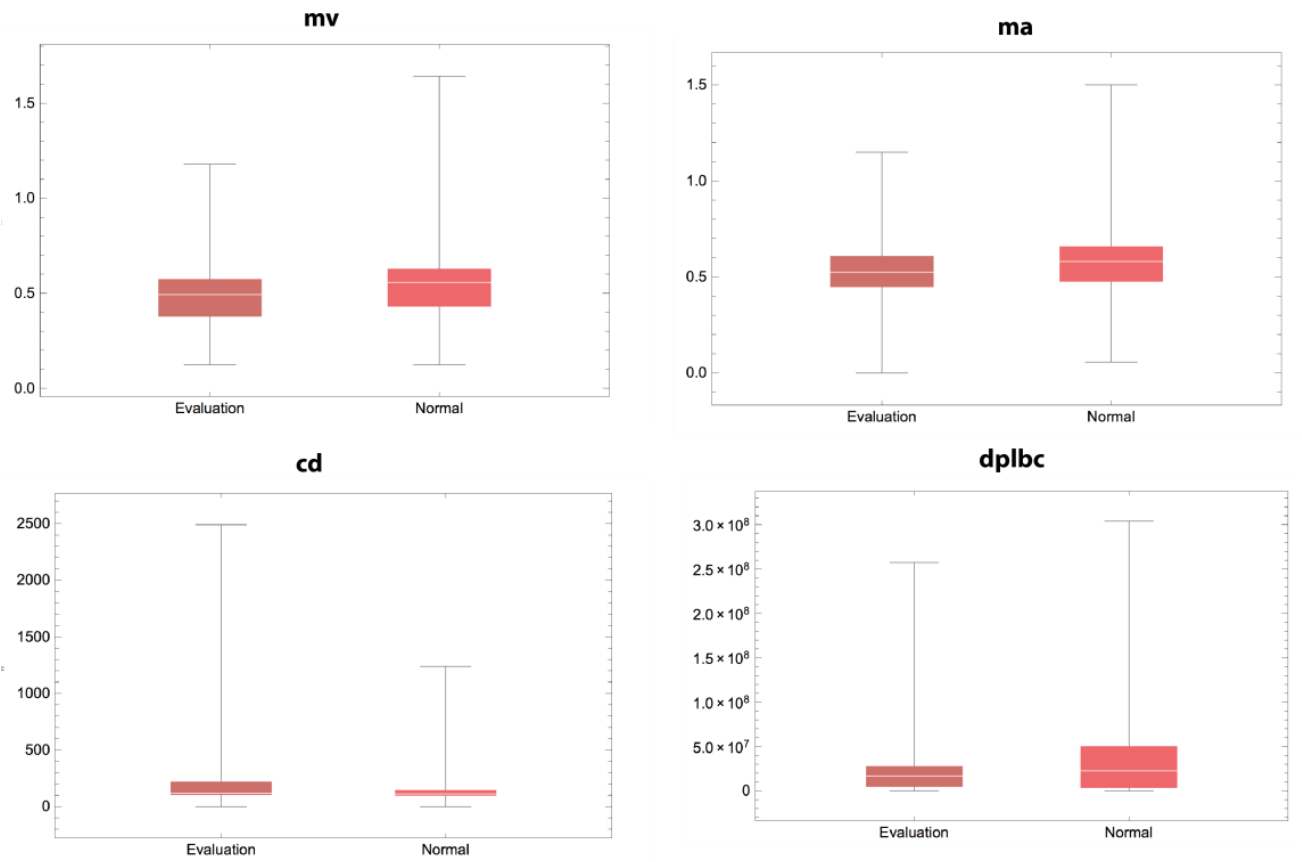

Fig. 2. Distributions of the data collected in the two different classes (Evaluation vs. Normal), in four different features: Mouse Velocity, Mouse Acceleration, Click Duration and Distance of the Pointer do the Line Between Clicks.

The selected of features characterize several aspects of interaction on normal and assessment lesson. However, this doesn't mean that they are all affected equally or 
that they are all affected at all when they have an assessment. A curious property of these features comparing the normal and the assessment lesson was that the mouse velocity and the mouse acceleration are lower and the click duration is higher in the assessment lesson. Figure 3 compares the histograms of mv, ma, and cd and evidences the differences between the two classes.
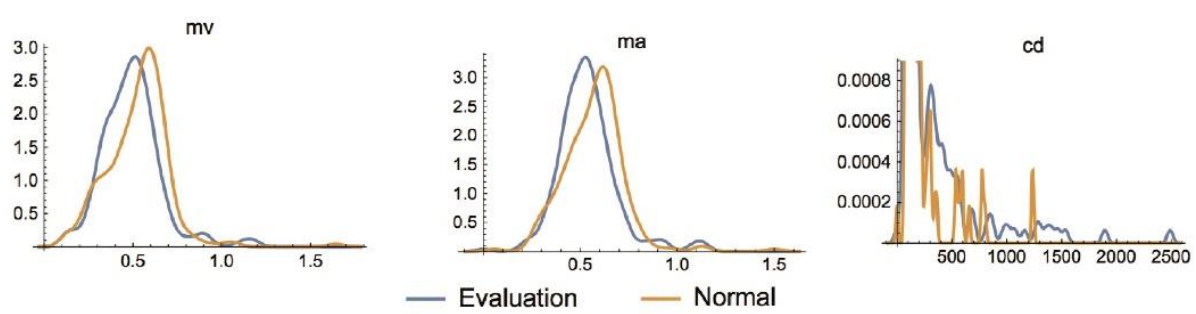

Fig. 3. Comparing mv, ma, and cd during the lessons.

From these studies we conclude that the most significantly affected features are mv, ma, cd, tbc, dbc, ddc, edbc, ssdbc, and dplbc. In all these features almost all students show statistically significant differences when comparing the two lessons.

\section{$4 \quad$ Preliminary Results}

During the two lessons, the monitoring system was used to assess the interaction of the students with the computer and to quantify their level of attentiveness, as well. To quantify attentiveness the following methodology was followed. Asides from capturing the interaction of the students with the computer, the monitoring system also registers the applications with which students are interacting. We analyzed all the applications used by all students and labelled each one of them as belonging to the task or not. We then quantify the amount of time that each student spends interacting with applications related to the task versus other applications.

Table 2 details the results of this quantification. Students have a clearly different attitude in the two lessons: when being evaluated, they spend more time interacting with task-related applications, a sign that they are more focused on the task. The results thus point out, not only, that it is possible to quantify student attentiveness in a non-intrusive way, but also that attention is higher when under evaluation. This was an expected conclusion but it nonetheless validates the proposed approach.

We also analyzed the correlation between the level of attentiveness and the score of the students in the task. There is a weak positive correlation $(0.41)$ between the two variables. We believe that this value is not higher for two reasons: (1) this class is mostly composed by "excellent" students and (2) the task was of average difficulty. Thus, there were not many differences in the scores. In future work we will study this relationship in more detail, namely in different classes and with tasks of different levels of difficulty. 


\begin{tabular}{|c|c|c|c|c|}
\hline \multirow{2}{*}{ Student } & \multicolumn{2}{|c|}{ Assessment } & \multicolumn{2}{c|}{ Normal } \\
\cline { 2 - 5 } & Total Time (s) & \% Time & Total Time (s) & \% Time \\
\hline T7110001 & 3156,22 & $67 \%$ & 690,20 & $31 \%$ \\
\hline T7110003 & 2416,01 & $55 \%$ & 1253,07 & $32 \%$ \\
\hline T7110005 & 2475,14 & $63 \%$ & 1807,26 & $40 \%$ \\
\hline T7110006 & 1571,11 & $36 \%$ & 494,07 & $19 \%$ \\
\hline T7110007 & 3177,28 & $53 \%$ & 701,26 & $30 \%$ \\
\hline T7110008 & 2492,51 & $58 \%$ & 783,33 & $26 \%$ \\
\hline T7110009 & 4264,73 & $72 \%$ & 2130,49 & $53 \%$ \\
\hline T7110010 & 3239,52 & $74 \%$ & 1451,13 & $40 \%$ \\
\hline T7110011 & 3845,71 & $71 \%$ & 737,86 & $20 \%$ \\
\hline T7110012 & 3581,57 & $58 \%$ & 157,73 & $30 \%$ \\
\hline T7110013 & 835,24 & $48 \%$ & 1588,86 & $40 \%$ \\
\hline
\end{tabular}

Table 2. Total time (seconds) devoted to the task and percentage of total time devoted to the task, while being assessed and while in a normal class, for each student.

\section{$5 \quad$ Discussion and Future Work}

It is imperative to mention that these features aim to quantify the student's work and estimate the level of attentiveness. These experiments allow drawing some interesting conclusions about students and their behavior during the assessment. However, this test was implemented in a good class with higher scores in all subjects. Yet, they react in different ways during normal or assessment lessons. We can see that they are more focused on the assessment lesson so they have a higher attention level in these situations. We also concluded that there are also significant differences in the interaction patterns when comparing the two classes.

In future work we will determine if there is a relationship between these two phenomena or if they are both dependent on other factors, such as the level of stress. We will also implement similar studies in other classes with overall worse scores and using tasks of different complexities. With this we will train classifiers that can estimate the level of attention of students, in real-time, to provide an important source of information for teachers to act accordingly and in a timely manner [15].

\section{Acknowledgements}

This work has been supported by COMPETE: POCI-01-0145-FEDER-007043 and FCT - Fundação para a Ciência e Tecnologia within the Project Scope: UID/CEC/00319/2013. 


\section{References}

1. Pimenta, A., Gonçalves, S., Carneiro, D., Riverola, F., Novais, P. (2014). Mental Workload Management as a Tool in e-Learning Scenarios.

2. Hwang, K., Yang, C. (2009). Automated Inattention and Fatigue Detection System in Distance Education for Elementary School Students. Journal of Educational Technology 12 pp. 22-35.

3. Esysench, M.W. (1992). Anxiety: The cognitive Perspective. Hove, England: Erlbaum.

4. Pimenta, A., Carneiro D., Neves, J., Novais P. (2015). A Neural Network to Classify Fatigue from Human-Computer Interaction. Neurocomputing. vol.172, pp. 413-426.

5. Olff, M. et al. (2005). The Psychobiology of PTSD: coping with trauma. Psichoneuroendocrinology 30, 974-982.

6. Oitzl, M.S. and Kloet, E.R. (1992). Selective corticosteroid antagonists modulate specific aspects of spatial orientation learning. Behav. Neurosci. 106, 62-71.

7. Sandi, C. and Rose, S.P. (1994). Corticosterone enhances log-term retention in one-dayold chicks trained in a weak passive avoidance learning paradigm. Brain. Res. 647, 106112.

8. Sandi, C. (1997). Experience-dependence facilitating effect of corticosterone on spatial memory formation in the water maze. Eur. J. Neurosci. 9, 637-642.

9. Roosendaal, B. and MAcGaugh, J.L.(1996). Amygdaloid nuclei lesions differentially affect glucorticoid-induced memory enhancement in a inhibitory avoidance. Task. Neurobiol. Learn. Mem. 65, 1-8.

10. Shors, T.J. Stressfull experience and Learning across Lifespan. Annu. Rev. Psychol. (in press).

11. McGaugh, J.L. (2004). The Amygdala Modulates the Consolidation of Memories of Emotionally arousing Experiences. Annu. Rev. Neurosci. 27, 1-28.

12. Bishop, S. (2008). Trait Anxiety and Impoverished Prefrontal Control of Attention. Nature Neuroscience 12, pp. 92-98.

13. van der Linden, D., Frese, M., Meijman, T.F. (2003). Mental Fatigue and the Control of Cognitive Processes: Effects on Perseveration and Planning. Acta Psychologica 113(1) pp. 45-65.

14. Carneiro, D., Novais, P., Pêgo, J.M., Sousa, N., Neves, J. (2015). Using Mouse Dynamics to Assess During Online Exams. Hybrid Artificial Intelligent Systems, vol. 9121, pp. 345356.

15. Lima, L., Novais, P., Costa, R., Cruz, J. B., \& Neves, J. (2010). Group decision making and Quality-of-Information in e-Health systems. Logic Journal of IGPL, jzq029.

16. Pimenta, A., Carneiro, D., Novais, P., \& Neves, J. (2013). Monitoring mental fatigue through the analysis of keyboard and mouse interaction patterns. In Hybrid Artificial Intelligent Systems (pp. 222-231). Springer Berlin Heidelberg. 